\title{
A Numerical Study of the Effect of Vortex-Induced Vibrations on a Circular Cylinder Mounted under Elastic Support
}

\section{Hugo Canilho ${ }^{1}$, Cristina Fael ${ }^{1}$, and José Páscoa ${ }^{2}$}

${ }^{1}$ CMADE - Centre of Building Materials and Technologies, University of Beira Interior, Portugal ${ }^{2}$ CMAST - Center for Mechanical and Aerospace Science and Technologies, University of Beira Interior, Portugal

\section{Abstract}

Vortex Induced Vibration (VIV) of structures is of practical interest to many fields of engineering. The particular case of a rigid cylinder mounted under elastic supports and constrained to oscillate in a single direction is modelled using OpenFOAM's two-dimensional Reynolds-averaged Navier-Stokes (RANS) equations with $k-\omega$ SST turbulence model. The model aimed for relativelly low Reynolds numbers ( $2500 \leq \mathrm{Re}$ $\leq 15000$ ) and the results were compared with Khalak and Williamson's experimental

Corresponding Author: Hugo Canilho hugo.canilho@ubi.pt

Received: 7 January 2020 Accepted: 21 April 2020 Published: 3 May 2020

Publishing services provided by Knowledge E

(c) Hugo Canilho et al. This article is distributed under the terms of the Creative Commons Attribution License, which permits unrestricted use and redistribution provided that the original author and source are credited.

Selection and Peer-review under the responsibility of the STARTCON19 Conference Committee.

\section{G OPEN ACCESS} results with the intent of also evaluating maximum amplitude to diameter ratio, $A / D$, achieving good agreement between both computational and experimental data. Lift and drag coefficients, $C_{L}$ and $C_{D}$ respectively, were also evaluated as well as frequency of oscillation, so the system could be kept in synchronization as what concerns the vortex shedding frequency and oscillating frequency $\left(\mathrm{f}_{s} \approx \mathrm{f}_{\text {osc }}\right)$. In this range of Reynolds numbers, as normalized velocity $\left(U^{*}\right)$ increased, several zones could be observed: The initial excitation zone, which jumps quickly to and upper branch zone at $U^{*} \approx 4.5$, moving to a lower branch zone at $U^{*} \approx 7$ and falling into desynchronization at $U^{*} \approx 11-13$.

\section{Introduction}

Vortex-induced vibrations (VIV) is considered by many an issue that can affect any structure and it has been studied by a lot of researchers in order to understand more deeply the phenomena. Having rigid cylinder mounted under elastic supports when inserted in a free stream and constrained to oscillate transversely to its direction is one of the most basic ways to understand VIV. One of the first studies on the topic was performed by Feng [1], where he conducted a detailed investigation of the vortex shedding frequency, displacement amplitude and the phase angle between the fluctuating pressure and the displacement signals of both circular and D-section cylinders. As a result of it, lift coefficients were made possible to be obtained, although, not by measurement but rather by integration. After some review documents on vortex shedding from oscillating bluff bodies [2, 3], Khalak and Williamson [4-6] did some 
research on the same topic as Feng [1], on low mass (around 1\% of what was done in his study) and damping systems, allowing them to test cylinder cases in laboratory in a more convenient way. In these systems there are four main zones of displacement of the cylinder. The initial excitation, the upper branch of the excitation, the lower branch and finally the desynchronization zone. As laboratory facilities evolved, more experiments were made available, and that is the case of the experiments made by Blevins and Coughran [7], where VIV forces were tested with one and two vibrating directions, with variable mass, damping and Reynolds number which was a major contribution to the understanding of VIV since amplitude, frequency, entrainment, and drag were reported in the paper.

This displacement was in more recent studies, harnessed to produce energy either when the fluid is water or air. Bernitsas et al. [8] created Vortex Induced Vibration Aquatic Clean Energy (VIVACE), a device that makes use of two different vortex induced motions phenomena, VIV forces and galloping to produce energy. Initially developed in 2008, it has been improved since to maximize displacement directly improving energy output [9-13]. As of wind energy harnessing, Chizfahm et al [14] used a different approach harnessing VIV, where the cylinder is constrained in one side only and it is free to move in both normal and transverse directions of the flow.

What concerns numerical approaches, Wanderley et al. [15] investigated the VIV phenomena by implementing a finite difference method (FDM) using slightly compressible Reynolds-Averaged Navier Stokes (RANS) equations with the k- $\varepsilon$ turbulence model. For high mass ratio the model was able to reproduce experimental data but for low mass ratios, such as the case of Khalak and Williamson [6], CFD failed to reproduce VIV amplitudes. Later in 2012, with a new study, that problem was solved with some adjustments to the model which allowed very good approximation to Khalak and Williamson's study [16].

Wu et al. [17] did some similar experiment to this one, although with Reynolds from 35000 to 130000 and using the 2D unsteady RANS equations (URANS) with the Spalart Allmaras turbulence model to simulate the flow around the spring mounted circular cylinder, where with the harnessing of VIV and galloping by introducing passive turbulence control (PTC) in the model, good results were obtained agreeing with experimental results even though the turbulence model was only a one equation model.

Hence, in the present work, the accuracy of the SST k- $\omega$ model when VIV forces are present in the domain is verified. 


\section{Vortex Induced Vibrations}

In general, the separation of the boundary layer occurs due to the adverse pressure gradient. This unfavorable pressure gradient is caused by the geometry of the body and occurs when the viscous forces are not sufficient to keep the flow adjacent to the wall, due to its minor contribution towards the inertial forces of the fluid moving around the surface.

The ratio between the viscous and inertial forces in a flow is represented by the dimensionless Reynolds Number, $\mathrm{R}_{e}$, given by Eq. (1).

$$
R_{e}=\frac{\rho U D}{\mu}
$$

with $\rho$ and $\mu$ being the density and the dynamic viscosity of the fluid, respectively, $D$ the characteristic length of the body and $U$ the average velocity in the undisturbed region of the flow.

The phenomenon of separation of the boundary layer of the body surface is governed by the Reynolds Number and all the characteristics of this separation, transition and wake have a relation with this dimensionless parameter.

For extremely low Reynolds values, the flow is entirely laminar and remains adjacent to the wall due to the dominance of viscous forces. As the Reynolds number increases, the inertial forces begin to prevail over the viscous ones so the flow can no longer withstand the adverse pressure gradient and the separation of the boundary layer with the body finally occurs. Different flow patterns occur in the disturbed regions due to the separation of the flow and the transition from the laminar to turbulent regime, according to the Reynolds Number.

The transition phenomenon and the classification of the regimes are important to better understand the flow patterns that will form in the wake region, which is the most important region in studies of flow around cylindrical bodies, since the fluctuations that occur in this region will reflect directly on the surface of the body and are responsible for causing vibrations, which will be studied in this work.

The wake region is composed by vortex generated on the surface that are dragged by the flow. The vortex shedding frequency, $f_{s}$, depends on the distance between the shear layers. The closer they are, the faster they interact with each other and the greater the vortex shedding frequency will be. The distance between these layers is associated with cylinder diameter and flow velocity so, the frequency of vortex emission is also associated with the Reynolds number of the flow and the point of separation of the vortex. The forces acting on the cylinder surface are then analysed for a better 
understanding of how the vibrations induced by the flow are evaluated. The force resulting from the interaction between the flow and the cylinder can be decomposed into two components: Drag force, $F_{D}$, which is the projected force in the longitudinal direction of the flow, and the lift force, $F_{L}$, which is the vertical component, normal to the flow.

When expressed in dimensionless terms of dynamic pressure of the fluid, that is, by the amount of kinetic energy that can be converted into pressure in cases of stagnation, we obtain the drag coefficients, $C_{D}$, and lift, $C_{L}$, given by Eq. (2) and (3), respectively.

$$
\begin{aligned}
C_{D} & =\frac{F_{D}}{0.5 \rho U^{2} D L} \\
C_{L} & =\frac{F_{L}}{0.5 \rho U^{2} D L}
\end{aligned}
$$

In these equations, $D L$ is the product of the diameter by the length of the cylinder, resulting in the projected area in the direction normal to the flow.

Another dimensionless parameter equally relevant to the present study, which also evolves with the Reynolds number is the Strouhal number, St, and its purpose is to relate the frequency of vortex emission with the geometric and flow characteristics, stated in Eq. (4).

$$
S t=\frac{f_{s} D}{U}
$$

where the vortex shedding frequency, $f_{s}$, is expressed in Hertz $[\mathrm{Hz}]$.

When a fluid flows past a structure, can cause a phenomenon referred to as flow induced vibration. This phenomenon can manifest itself in some ways. Among these possible types of excitation, the most frequent in cylindrical bodies is the Vibration Induced Vibration, theme in this work.

Thus, given a cylinder subject to the incident flow, the velocity of this flow can be varied so that the frequency of vortex shedding also varies, and consequently, the fluctuation of the pressure field around the cylinder. As the resulting forces acting on the body are nothing more than the integration of the pressure field on the surface, thereof, this temporal variation of the vertical lift force causes an excitation with a defined frequency, the same as that of vortex shedding.

One may think that this variation in the pressure field on the cylinder surface occurs so that there is an excitation towards the flow and another in the direction transverse to it, in the vertical direction. This effectively happens, but with different frequencies for the same flow condition. The frequency of drag variation is approximately twice as high as the lift force frequency since the lift force varies from its positive maximum point 
to the negative maximum point for each vortex emission cycle while the drag force alternates between the maximum and minimum values for each vortex emitted, even though it changes direction.

Once these phenomena that cause forces in the system are understood, it is still necessary to understand the characteristics of the structure responsible for responding to these excitations and what is the cause of the amplitudes of the vibrations. Thus, the equation of motion representing the vortex-induced vibrations in a cylinder oscillating in the y direction (normal to the flow) is expressed by Eq. (5).

$$
m \ddot{y}+c \dot{y}+k y=F
$$

where $\mathrm{m}$ is the mass of the oscillating structure, $c$ is the damping constant of the system, $k$ is the stiffness constant of the structure, $F$ is the excitation force in the transverse direction e $\ddot{y}, \dot{y}$ and $y$, are the acceleration, velocity, and position in the $y$ direction. In a regime where the natural frequency of the structure is synchronized with the excitation force of the structure, a good approximation of the force, $F$, over time is given by Eq. (6).

$$
F(t)=F_{0} \sin (\omega t+\phi)
$$

where $F_{0}$ is the magnitude of the force caused by the flow, $\omega$ is the natural oscillation frequency of the body given in radians per second, and $\varphi$ is the phase angle between the excitation and the displacement of the cylinder and $t$ is the time. Since the systems subject to VIV can be modelled as harmonic systems without impairing the physical representation of the problem, the system response can be given Eq. (7).

$$
y(t)=y_{0} \sin (\omega t)
$$

In dynamic systems that are governed by Equation (5) without damping, its angular velocity can be expressed Eq. (8)

$$
\omega=2 \pi f_{N}=\sqrt{K / m}
$$

where $f_{N}$ is the frequency of vortex shedding, $K$ is the spring stiffness and $m$ is the mass of the system. In order to obtain greater accuracy in the comparisons between cases where the operating conditions are different, whether the frequency or any other type of parameters, it is important to carry out analyses under non-dimensional conditions. Table 1 defines the most used parameters in this type of analysis, where $m_{d}$ is the displaced mass, and $c_{c r}$ is the critical damping of the system.

Given these parameters and by knowing how to calculate them, it is possible to understand the behaviour of this system. 
TABLE 1: Non-dimensional parameters of VIV systems.

$\mathbf{A}^{*}$
$\mathbf{U}^{*}$
$\mathbf{m}^{*}$
f*
?e

$\operatorname{Re}$

$y_{0} / D$
$U / f_{N} D$
$m / m_{d}=m / \pi \rho D^{2} L / 4$
$f / f_{N}$
$c / c_{c r}=c / 2 \sqrt{k m}$
$\rho U D / v$

\section{Problem Description}

In the present study, the effect of vortex induced vibrations in a single circular cylinder is investigated under Reynolds numbers of $2500 \leq R e \leq 15000$. The simulations presented in this study are obtained through the open source CFD C++ tool OpenFOAM.

The flow is assumed to be two-dimensional, unsteady, incompressible and as stated previously by the Reynolds number, turbulent.

The continuity equation as well as momentum equation (Eq. (9) and (10), respectively) are solved using the OpenFOAM software which uses finite volume method (FVM).

$$
\begin{gathered}
\frac{\partial\left(u_{i}\right)}{\partial x_{i}}=0 \\
\rho\left(\frac{\partial\left(u_{i}\right)}{\partial t}+\frac{\partial\left(u_{j} u_{i}\right)}{\partial x_{j}}\right)=-\frac{\partial p}{\partial x_{i}}+\frac{\partial}{\partial x_{j}}\left[\mu\left(\frac{\partial u_{i}}{\partial x_{j}}+\frac{\partial u_{j}}{\partial x_{i}}\right)-\rho \overline{u_{i}^{\prime} u_{j}^{\prime}}\right]
\end{gathered}
$$

where $u$ and $u^{\prime}$ are the mean and fluctuating velocity components, $\rho$ is density, $p$ is pressure, $x$ is coordinate and the subscripts $i$ and $j$ denote the directions in Cartesian coordinates. The last term $-\rho \overline{u_{i}^{\prime} u_{j}^{\prime}}$ is the Reynolds stress tensor, which needs modelling in order to solve Eq. (10). To do so, the k- $\omega$ SST model, which was developed by Menter [18] was chosen. It is a model that shares features of the $k-\varepsilon$ and $k-\omega$ models, as it results from a combination of both. This model becomes a $k-\varepsilon$ model in the outer region of the boundary layer, while in the inner part it becomes a $k-\omega$ model. Regarding the transport equations, the $k-\omega$ SST model is similar to the $k-\omega$ model but includes its own characteristics. The turbulent kinetic energy, $k$, and the specific dissipation rate, $\omega$, are obtained by Eq. (11) and (12), respectively.

$$
\begin{gathered}
\frac{\partial}{\partial t}(\rho k)+\frac{\partial}{\partial x_{i}}\left(\rho k u_{i}\right)=\frac{\partial}{\partial x_{j}}\left(\Gamma_{k} \frac{\partial k}{\partial x_{j}}\right)+G_{k}-Y_{k}+S_{k} \\
\frac{\partial}{\partial t}(\rho \omega)+\frac{\partial}{\partial x_{i}}\left(\rho \omega u_{i}\right)=\frac{\partial}{\partial x_{j}}\left(\Gamma_{\omega} \frac{\partial \omega}{\partial x_{j}}\right)+G_{\omega}-Y_{\omega}+D_{\omega}+S_{\omega}
\end{gathered}
$$

In these equations, $G_{k}$, represents the production of turbulent kinetic energy due to velocity gradients, $G_{\omega}$ represents the production of $\omega, \Gamma_{k}$ and $\Gamma_{\omega}$ represent the effective 
diffusivity of $k$ and $\omega$, respectively. $Y_{k}$ and $Y_{\omega}$ represent the dissipation of $k$ and $\omega$ due to the turbulence. $D_{\omega}$ represents the term of crushed diffusion.

The constants of this model take the values given in Table 2 .

TABLE 2: k- $\omega$ SST model constants.

\begin{tabular}{|c|c|c|c|c|c|c|c|}
\hline $\boldsymbol{\sigma}_{\boldsymbol{k}, \mathbf{1}}$ & $\boldsymbol{\sigma}_{\boldsymbol{\omega}, \mathbf{1}}$ & $\boldsymbol{\sigma}_{\boldsymbol{k}, \mathbf{2}}$ & $\boldsymbol{\sigma}_{\boldsymbol{\omega , \mathbf { 2 }}}$ & $\boldsymbol{a}_{\mathbf{1}}$ & $\boldsymbol{\beta}_{\boldsymbol{i , 1}}$ & $\boldsymbol{\beta}_{\boldsymbol{i}, \mathbf{2}}$ & $\boldsymbol{\sigma}_{\boldsymbol{\infty}}^{*}$ \\
\hline 1.176 & 2.0 & 1.0 & 1.168 & 0.31 & 0.075 & 0.0828 & 1.0 \\
\hline $\boldsymbol{\sigma}_{\boldsymbol{\infty}}$ & $\boldsymbol{\sigma}_{\mathbf{0}}$ & $\boldsymbol{\beta}_{\boldsymbol{\infty}}^{*}$ & $\boldsymbol{R}_{\boldsymbol{\beta}}$ & $\boldsymbol{R}_{\boldsymbol{k}}$ & $\boldsymbol{R}_{\boldsymbol{\omega}}$ & $\boldsymbol{\zeta}^{*}$ & $\boldsymbol{M}_{\mathbf{t} 0}$ \\
\hline 0.52 & $1 / 9$ & 0.09 & 8.0 & 6.0 & 2.95 & 1.5 & 0.25 \\
\hline
\end{tabular}

\subsection{Computational Domain}

The computational domain considered in the present study is shown in Fig. 1, following the work of Wu et al. [17] and Wanderley et al. [15, 16]. A two-dimensional computational domain was investigated for numerical analysis. The size of the computational domain, shown in Fig. 1 was $400 \times 200$ points, where the external boundary was 120 diameters from the body surface, following the work of Wanderley et al. $[15,16]$, with a steady twodimensional and uniform flow, sufficient to predict the flow mechanism of the present problem.

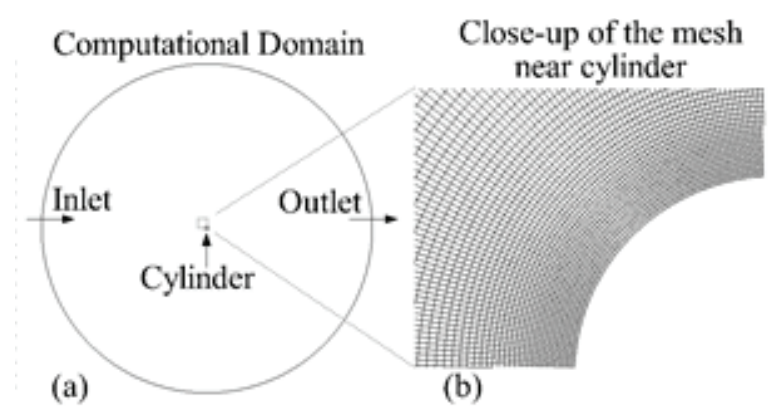

Figure 1: (a) Computational domain; (b) Close-up of the mesh near the cylinder.

The origin of the coordinate system was located at the center of the oscillating cylinder in the beginning of the numerical analysis, with $x$ and $y$ in the streamwise and normal directions, respectively. The dimensions of the channel enclosing the cylinder to simulate the mainstream flow were sufficiently large that the formation of flow vortices near the body was not affected by the boundaries of the channel.

The whole computational domain is divided into two zones, viz., fixed mesh zone and dynamic mesh zone. These two zones are separated by a circular interface 10 diameters away from the cylinder. As of the boundary conditions, the flow comes from left to right, so the inlet is located on the left side of the domain, where the entry velocity is specified, 
and the outlet is located in the right side. The cylinder is treated as a dynamic wall, with six degrees of freedom, allowing it to move in the direction transverse to the flow, and blocking it from moving either in the flow direction or to rotate. As it was already stated, the cylinder is mounted under elastic supports, that means that a spring and damper are included in the setup and kept constant as the velocity increases (Also increasing Reynolds Number).

\section{Results and Discussion}

\subsection{Grid Independence study}

To verify the influence of the mesh size on the numerical results, three different grids were tested for Reynolds number equal to 1000 . The first grid tested had $400 \times 150$ points (coarse grid), the second grid had $400 \times 200$ points, and finally the third and finer grid had $400 \times 250$ points. The effect of the grid refinement is negligible as no difference is observed between the results.

Therefore, the grid with $400 \times 200$ points proved to be good enough for the present investigation and was used in the entire investigation. Table 3 summarizes the Strouhal number, drag, and lift coefficients obtained for Reynolds number equal to 1000 and the three different grid refinement.

TABLE 3: Strouhal number, lift, and drag coefficients obtained for three different mesh refinements and $\mathrm{Re}=1000$.

\begin{tabular}{|l|c|c|c|c|}
\hline Re & $\mathbf{C}_{D}$ & $\mathbf{C}_{L}$ & $\mathbf{S t}$ & Grid \\
\hline \multirow{2}{*}{1000} & 0.965 & 0.238 & 0.191 & $400 \times 150$ \\
\hline & 0.961 & 0.222 & 0.193 & $400 \times 200$ \\
\hline & 0.961 & 0.222 & 0.193 & $400 \times 250$ \\
\hline
\end{tabular}

\subsection{Numerical Results}

The objective of the present investigation is to duplicate in accurate and reliable way, the benchmarking experimental results obtained by Khalak and Williamson [4]. Thus, the experimental setup considered throughout the numerical simulation was the same as the experimental and also the same mass ratio of $\mathrm{m}^{*}=2.4$, damping ratio of $\zeta=0.0045$, and reduced velocity varying from 2.5 to 14 , corresponding to Reynolds number variation from 2000 to 12000. 
Fig. 2a shows the comparison between the numerical results obtained in the present work for the amplitude of oscillation as a function of reduced velocity and experimental data from Khalak and Williamson [4]. In this numerical simulation, all results were obtained by increasing the reduced velocity step by step. As reduced velocity increases, the amplitude of oscillation increases and reaches a maximum and then starts decreasing. The agreement between the numerical results obtained in the present work and the experimental results obtained in Khalak and Williamson [4] is good, considering the struggles that numerical simulations have to reproduce experimental results on VIV.

Fig. 2b presents the vortex shedding frequency of the fixed cylinder, the frequency of oscillation of the moving cylinder as functions of reduced velocity. The frequency is approximately constant inside the lock-in region and increases with reduced velocity outside the lock-in region. In Fig. 2a, the point of maximum amplitude of oscillation corresponds to a reduced velocity of 5.2 and a frequency ratio of $f=f_{n}=0.88$, see Fig. $2 b$. This means that, at the peak, the frequency of vortex shedding is lower than the natural frequency of the structure (in air). As the velocity of the flow is increased, the amplitude decreases continuously up to a reduced velocity of 5.5 and frequency ratio of $f=f_{n}=0.98$. The next point corresponds to a reduced velocity of 6.0 and a frequency ratio of $f=f_{n}=1.06$. Now, the frequency of vortex shedding is greater than the natural frequency of the structure, and there is a jump in amplitude shown in Fig. 2a. In the subsequent points, increasing the reduced velocity makes the frequency ratio remain approximately constant up to reduced velocity equal to 10.5 .

Beyond this point, synchronization stops, the amplitude decreases drastically, and the frequency of vortex shedding returns to the Strouhal frequency. In Fig. 2b, the frequency of vortex shedding is given by Eq. (13).

$$
f_{N}=\sqrt{K /\left(m+m_{a}\right)}
$$

where

$$
m_{a}=\rho\left(\pi D^{2} / 4\right) C_{a}
$$

and

$$
C_{a}=1
$$

Here, $D$ is the cylinder diameter and $C_{a}$ is a constant.

Fig. 3a was obtained for reduced velocities $U^{*}=2.5$ at the initial branch $\left(U^{*}<4.0\right)$ of the amplitude of oscillation curve of Fig. 2a. Fig. 3a presents the vorticity field around the cylinder and Fig. $3 \mathrm{~b}$ presents the time traces of the displacement of the cylinder. 
(a)

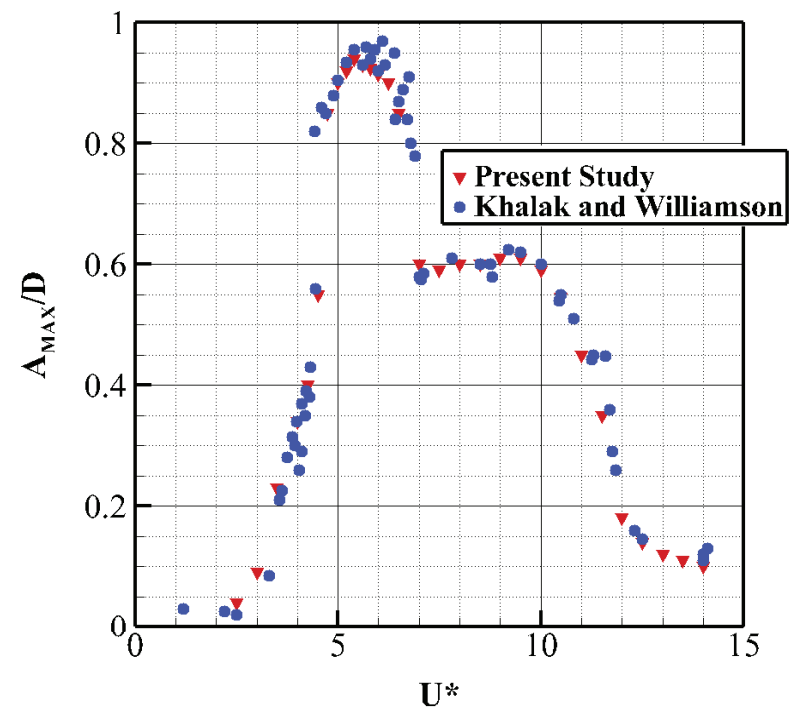

(b)

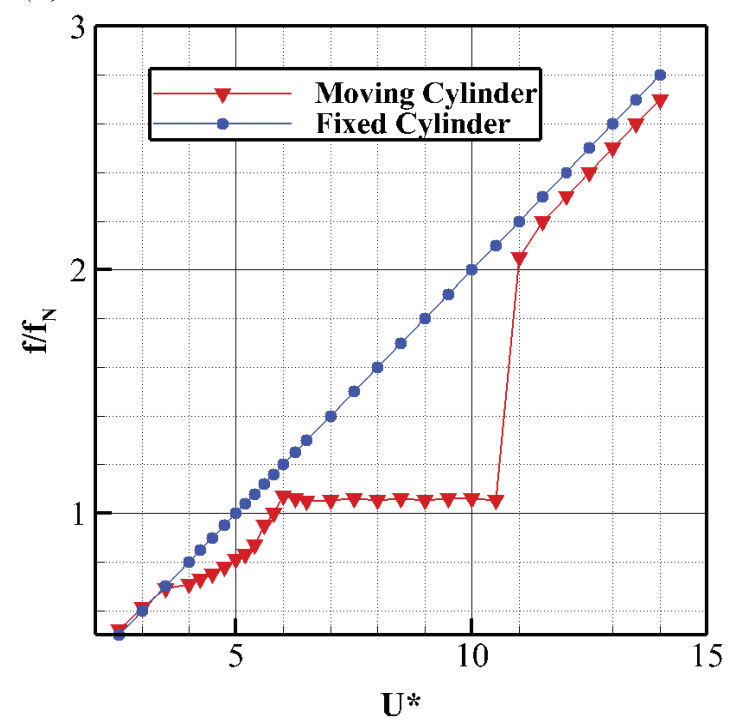

Figure 2: Results for the oscillating of a circular cylinder as a function of reduced velocity: (a) amplitude of displacement, and (b) frequency of oscillation.

Fig. $3 \mathrm{~b}$ shows that the displacement of the cylinder is almost inexistent to this velocity ranges. Fig. 3a shows that one vortex is shed in each half cycle of oscillation, which is compatible with the $2 \mathrm{~S}$ mode of Williamson and Roshko [19] for the initial branch.

Fig. $3 c$ represents the results for reduced velocities $U^{*}=5.5$ at the upper branch $\left(4.0<U^{*}<6.5\right)$ of the amplitude of oscillation curve of Fig. 2a. Fig. 3c shows that two vortices are shed in each half cycle of oscillation, which is compatible with the $2 \mathrm{P}$ mode, with the second vortex of each pair being much weaker than the first one. Fig. $3 d$ shows that the displacement ratio of the cylinder is almost equal to 1 , the maximum 
value for displacement of the cylinder, achieving good agreement with results from Khalak and Williamson [4].

Fig. 3e was obtained for reduced velocities $U^{*}=8.0$ at the lower branch $\left(6.0<U^{*}<10.5\right)$ of the amplitude curve of Fig. 2a. Fig. 3e presents the vorticity field around the cylinder while Fig. $3 f$ presents the time traces for the displacement of the cylinder. It shows that two vortices are shed in each half cycle of oscillation, which is compatible with the $2 \mathrm{P}$ mode of Williamson and Roshko [19] the lower branch.
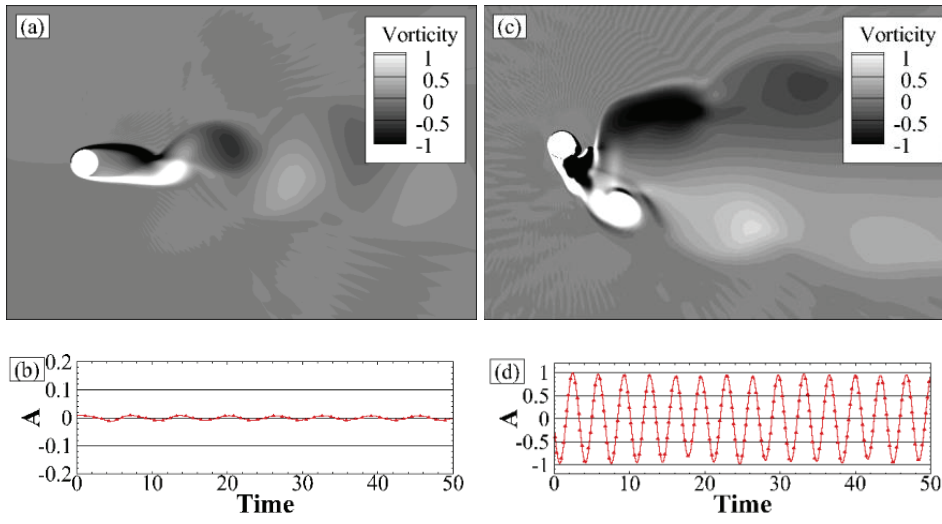
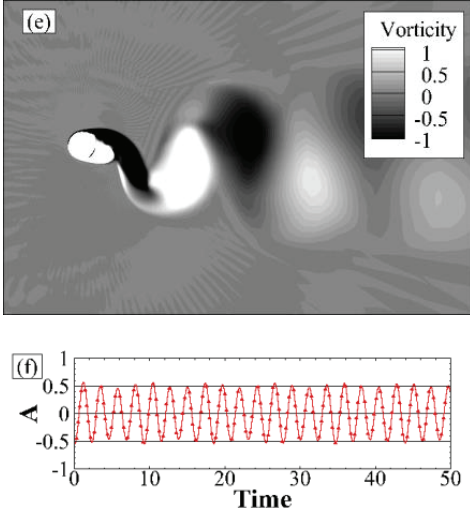

Figure 3: Results obtained for the vibrating circular cylinder of vorticity (top) and amplitude ratio (bottom) for: (a-b) $U^{*}=2.5$; (c-d) $U^{*}=5.5$; and $(e-f) U^{*}=8.0$.

\section{Conclusion}

The present work shows good agreement between the numerical simulations, which used the k- $\omega$ SST turbulence model, and experimental results obtained by Khalak and Williamson [4], for the specific case of an elastically mounted cylinder under vortex induced vibration effects.

The OpenFOAM code proved to be reliable when working with the $k-\omega$ SST turbulence model, allowing for other approach of the VIV enhancement for different geometries, in future research.

The numerical results show that the frequency of vortex shedding is synchronized with the natural frequency of the cylinder, for a deep range of reduced velocities $\left(5<U^{*}<10.5\right)$ whiles synchronized. The cylinder reaches maximum amplitude ratios of nearly one, for $U^{*}=5.2$, and over 0.6 for most of the synchronization zone.

In reduced velocities higher than 10.5, the synchronization stops and makes the oscillation of the body almost null. The frequency of vortex shedding, from this point on, is approximately the Strouhal frequency once again, as it was from $U^{*}<5$ 


\section{Acknowledgements}

This work was supported with Portuguese national funds by FCT - Foundation for Science and Technology within the Grant ERANETMED/0004/2014 (Project ID eranetmed_ nexus-14-044) and the UID/ECI/04082/2013 project, and, within the research unit CMADE, Centre of Materials and Building Technologies (CIVE-Central Covilhã-4082), University of Beira Interior, Portugal.

\section{References}

[1] C. C. Feng, "The measurement of vortex induced effects in flow past stationary and oscillating circular and D-section cylinders," University of British Columbia, Vancouver, Canada, 1968.

[2] T. Sarpkaya, "Vortex induced oscillations - A selective review," J. Appl. Mech., 46, pp. 241-258, (1979).

[3] P. W. Bearman, "Vortex shedding from oscillating bluff bodies," Annu. Rev. Fluid Mech., 16, pp. 195-222, (1984).

[4] A. Khalak and C.H.K. Williamson, "Dynamics of a hydroelastic cylinder with very low mass and damping," J. Fluids Struct., 10, pp. 455-472, (1996).

[5] A. Khalak and C.H.K. Williamson, "Fluid forces and dynamics of a hydroelastic structure with very low mass damping," J. Fluids Struct., 11, pp. 973-982, (1997).

[6] A. Khalak and C.H.K. Williamson, "Motions, Forces and Mode Transitions in VortexInduced Vibrations At Low Mass-Damping," J. Fluids Struct., 13, no. 7-8, pp. 813-851, (1999).

[7] R. D. Blevins and C. S. Coughran, "Experimental Investigation of Vortex-Induced Vibration in One and Two Dimensions With Variable Mass, Damping, and Reynolds Number," J. Fluids Eng., 131, no. 10, p. 101202, (2009).

[8] M. Bernitsas, K. Raghavan, Y. Ben-Simon, and E. Garcia, "VIVACE (Vortex Induced Vibration Aquatic Clean Energy): A New Concept in Generation of Clean and Renewable Energy From Fluid Flow," J. Offshore Mech. Arct. Eng., 130, no. 4, p. 41101, (2008).

[9] J. Lee, C. Chang, N. Xiros, and M. Bernitsas, "Integrated power take-off and virtual oscilator system for the vivace converter: Vck system identification," Proc. ASME 2009 Int. Mech. Eng. Congr. Expo., IMECE 2009, pp. 1-7, (2009). 
[10] C. Chang, R. Ajith Kumar, and M. Bernitsas, "VIV and galloping of single circular cylinder with surface roughness at $30000 \leq \operatorname{Re} \leq 120000$," Ocean Eng., 38, no. 16, pp. 1713-1732, (2011).

[11] C. Chang and M. Bernitsas, "Hydrokinetic Energy Harnessing Using the Vivace Converter With Passive Turbulence Control," in Proc. ASME 2011 Int. Conf. Ocean. Offshore Arct. Eng., 2011, pp. 1-10.

[12] C. Chang and M. Bernitsas, "Envelope of power harvested by a single-cylinder vivace converter," in Proc. ASME 2015 34th Int. Conf. Ocean. Offshore Arct. Eng., 2015.

[13] E. Kim and M. Bernitsas, "Performance prediction of horizontal hydrokinetic energy converter using multiple-cylinder synergy in flow induced motion," Appl. Energy, 170, pp. 92-100, (2016).

[14] A. Chizfahm, E. Azadi Yazdi, and M. Eghtesad, "Dynamic modelling of vortex induced vibration wind turbines," Renew. Energy, 121, no. 121, pp. 632-643, (2018).

[15] J. Wanderley, G. Souza, S. Sphaier, and C. Levi, "Vortex-induced vibration of an elastically mounted circular cylinder using an upwind TVD two-dimensional numerical scheme," Ocean Eng., 35, pp. 1533-1544, (2008).

[16] J. Wanderley, S. Sphaier, and C. Levi, "A two-dimensional numerical investigation of the hysteresis effect on vortex induced vibration on an elastically mounted rigid cylinder," J. Offshore Mech. Arct. Eng., 134, p. 21801, (2012).

[17] W. Wu, M. M. Bernitsas, and K. Maki, "RANS Simulation Versus Experiments of Flow Induced Motion of Circular Cylinder With Passive Turbulence Control at 35,000 \&lt; RE \&lt; 130,000," J. Offshore Mech. Arct. Eng., 136, no. 4, p. 041802, (2014).

[18] F. R. Menter, "Two-equation eddy-viscosity turbulence models for engineering applications," AIAA J., 32, no. 8, pp. 1598-1605, (1994).

[19] C. H. K. Williamson and A. Roshko, "Vortex formation in the wake of an oscillating cylinder," J. Fluids Struct., 2, no. 4, pp. 355-381, (1988). 